Portland State University

PDXScholar

3-15-1996

\title{
Efficient Buffer Allocation Scheme for Bursty Traffic in ATM Networks using Fast Reservation Protocol
}

Nikhil Mukund Deshpande

Portland State University

Follow this and additional works at: https://pdxscholar.library.pdx.edu/open_access_etds

Part of the Electrical and Computer Engineering Commons Let us know how access to this document benefits you.

\section{Recommended Citation}

Deshpande, Nikhil Mukund, "Efficient Buffer Allocation Scheme for Bursty Traffic in ATM Networks using Fast Reservation Protocol" (1996). Dissertations and Theses. Paper 5237.

https://doi.org/10.15760/etd.7110

This Thesis is brought to you for free and open access. It has been accepted for inclusion in Dissertations and Theses by an authorized administrator of PDXScholar. Please contact us if we can make this document more accessible: pdxscholar@pdx.edu. 


\section{THESIS APPROVAL}

The abstract and thesis of Nikhil M. Deshpande for the Master of Science in Electrical and Computer Engineering were presented March 15, 1996, and accepted by the thesis committee and the department.

COMMITTEE APPROVALS:

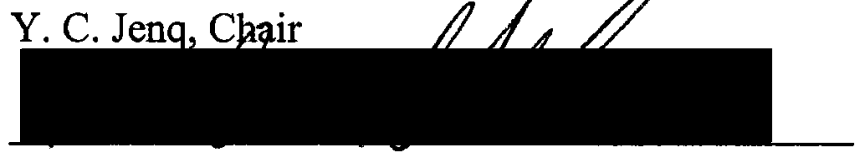

Michael A. Driscoll

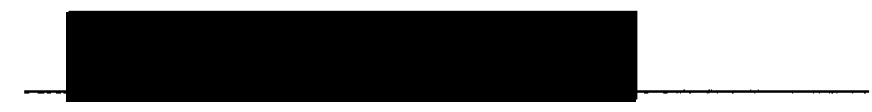

K. W. Tam

Representative of the Office of Graduate Studies

DEPARTMENT APPROVAL:

Rolf Schaumann, Chair

Department of Electrical Engineering

$* * * * * * * * * * * * * * * * * * * * * * * * * * * * * * * * * * * * * * * * * * * * * * * * * * * * * * * * * * * * * * * * * *$

ACCEPTED FOR PORTLAND STATE UNIVERSITY BY THE LIBRARY by on 4 tene 1996 


\begin{abstract}
An Abstract of the thesis of Nikhil M. Deshpande for the Master of Science in Electrical and Computer Engineering presented on March 15, 1996.

Title: Efficient Buffer Allocation Scheme for Bursty Traffic in ATM Networks using Fast Reservation protocol
\end{abstract}

One of the major problems in the realization of ATM is traffic management, in particular, the resource allocation for an efficient network. Many approaches to the bandwidth management problems [2] and buffer management problems have been proposed. Boyer [10] suggested a "Fast Bandwidth Reservation" protocol, while Turner [2] applied the same technique to the buffer allocation for the bursty traffic. In this thesis, we extend Turner's scheme in order to make it more efficient with respect to buffer allocation. We define a new parameter "Reduction Factor", which has the potential of saving a significant amount of buffer space in a commonly shared pool of buffers. It is observed that the amount of savings depends upon the cell loss probability (CLP) bound on the network, Source Activity Probability (SAP) and number of connections supported by the network $(\mathrm{N})$ at particular instant. The performance of the scheme is evaluated under various QoS requirements. It is seen that this scheme leads to approximately $38 \%$ of savings in the buffer space under certain circumstances. For supporting multimedia services, we also employ the scheme of partitioning 
the total buffer space. The results show that it leads to $10 \%$ less savings as compared to single buffer scheme under certain conditions. As usage policing is very critical in this scheme, we outline a suitable mechanism and hardware implementation for Usage Parameter Control. The algorithm for acceptance of virtual circuit in the network is also described. 
by

NIKHIL M. DESHPANDE

A thesis submitted in partial fulfillment of the requirements for the degree of

\author{
MASTER OF SCIENCE \\ in \\ ELECTRICAL AND COMPUTER ENGINEERING
}

Portland State University

1996 


\section{ACKNOWLEDGMENT}

I wish to express my sincere thanks to Dr. Y. C. Jenq for his guidance and support throughout the course of this work and my academic life. I would also like to thank Dr. Mike Driscoll from whose knowledge I benefited a lot. I wish to thank Shirley Clark, Laura Riddell and Ellen Wack of Dept. of Electrical Engineering at Portland State University.

I wish to express my deepest gratitude to my parents and my sister for their love and encouragement.

Last but not the least, I sincerely appreciate the financial assistance of the Department of Electrical Engineering at Portland State University. 


\section{TABLE OF CONTENTS}

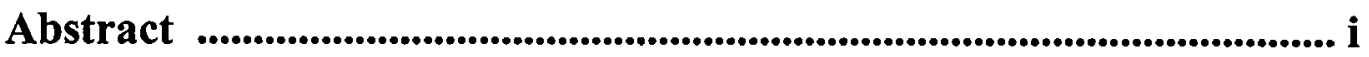

Acknowledgments ................................................................................................. iii

Table of Contents ................................................................................................. iv

Chapter 1 Introduction To ATM ........................................................ 1

1.1 ATM As a Technology ............................................. 1

1.2 Traffic Management and Related Issues in ATM 2

1.3 Objectives of ATM ................................................... 5

1.4 Review of Related Work .......................................... 6

1.5 Fast Buffer Reservation Protocol ........................... 7

1.6 Overview of the Thesis ............................................. 9

Chapter 2 Proposed Buffer Allocation Scheme for Bursty Traffic .11

2.1 Descriptions and Mathematical Formulations ... 11

2.2 Virtual Circuit Acceptance Algorithm ................... 14

2.3 Parameter Engineering ….............................................. 15

2.3.1 Reduction Factor ........................................... 15

2.3.2 Cell Loss Probability Threshold .............. 16 
Chapter 3 Performance Analysis of the Proposed Scheme 18

3.1 Performance of Single Buffer Scheme ................. 18

3.2 Usage Policing Mechanism .................................... 20

3.3 Inadequacies of Single Buffer Scheme ................ 26

Chapter 4 Partitioned Buffer Allocation Scheme ............................... 29

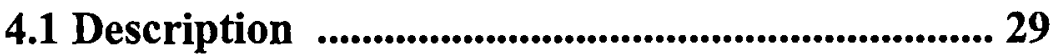

4.2 Performance Comparison of Partitioned Buffer

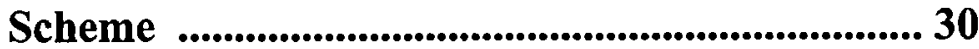

4.2.1 Single Buffer with CLP bound of

$1.5 \mathrm{E}-4$ 30

4.2.2 Single Buffer with CLP bound of

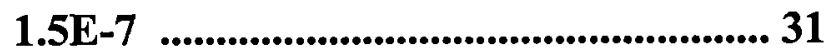

4.2.3 Partitioned Buffer with two independent service class space

4.3 Results of Comparison ....................................... 31 
Chapter 5 Conclusion And Research Findings ................................ 34

5.1 Discussion .................................................................. 34

5.2 Important Research Findings ................................ 34

5.3 Some Drawbacks of the Scheme and Solutions.. 35

5.4 Future Research .......................................................... 36

Appendix A Glossary ......................................................................................... 38

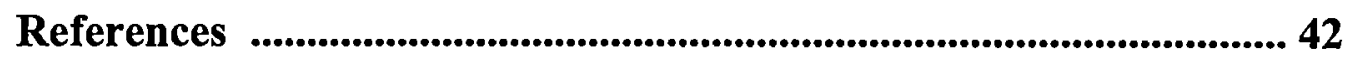




\section{CHAPTER 1}

\section{Introduction to ATM}

\subsection{ATM As a Technology}

Broadband Integrated Services Digital Networks (B-ISDN) are designed to support the integration of both non-real time traffic (e.g. data file transfer) and real time traffic (e.g. voice and video interactive services) over the same medium. The Asynchronous Transfer Mode (ATM) solution has been recommended by the ITU [1] to be the transport method of the B-ISDN networks. ATM is a cell based fast packet switching technology in which the information from different sources is chopped into small packets (ATM cells) with the same length (53 bytes) and transmitted over a single broadband network. The major advantages of this architecture include [3][4]:

A. Flexibility in accommodating existing and future unknown applications.

B. Simple multiplexing and switching.

C. Potential for statistical multiplexing to save bandwidth and other resources.

Because of its high bandwidth switching capability, ATM is being applied in both local area and wide area communication networks. Researchers are considering the possibility of applying ATM to existing networks like 
Internet (IP over ATM Group) or Local Area Networks ( ATM Interface at MAC sub-layer) [6].

ATM handles both connection-oriented traffic directly or through adaptation layers, or connectionless traffic through the use of adaptation layers. ATM virtual connections may operate at either a Constant Bit Rate (CBR) or a Variable Bit Rate (VBR). Each ATM cell sent into the network contains the addressing information that establishes a virtual connection from origination to destination. All cells are then transferred over this virtual connection. ATM provides either Permanent or Switched Virtual Connections (PVCs or SVCs). ATM is asynchronous because the transmitted cells need not be periodic as time slots of data.

ATM offers the potential to standardize on one network architecture defining multiplexing and switching method, with SONET/STM providing the basis for physical transmission standard for very high speed rates [16] [17]. ATM also supports multiple QoS (Quality of Service) classes for differing application requirements on delay and loss performance. Thus the vision of ATM is that an entire network can be constructed using ATM and ATM Adaptation Layers (AALs) switching and multiplexing principles to support a 
wide range of all services, such as voice, video, packet data, imaging and circuit emulation. Fig. 1.1 shows an example of an ATM application.

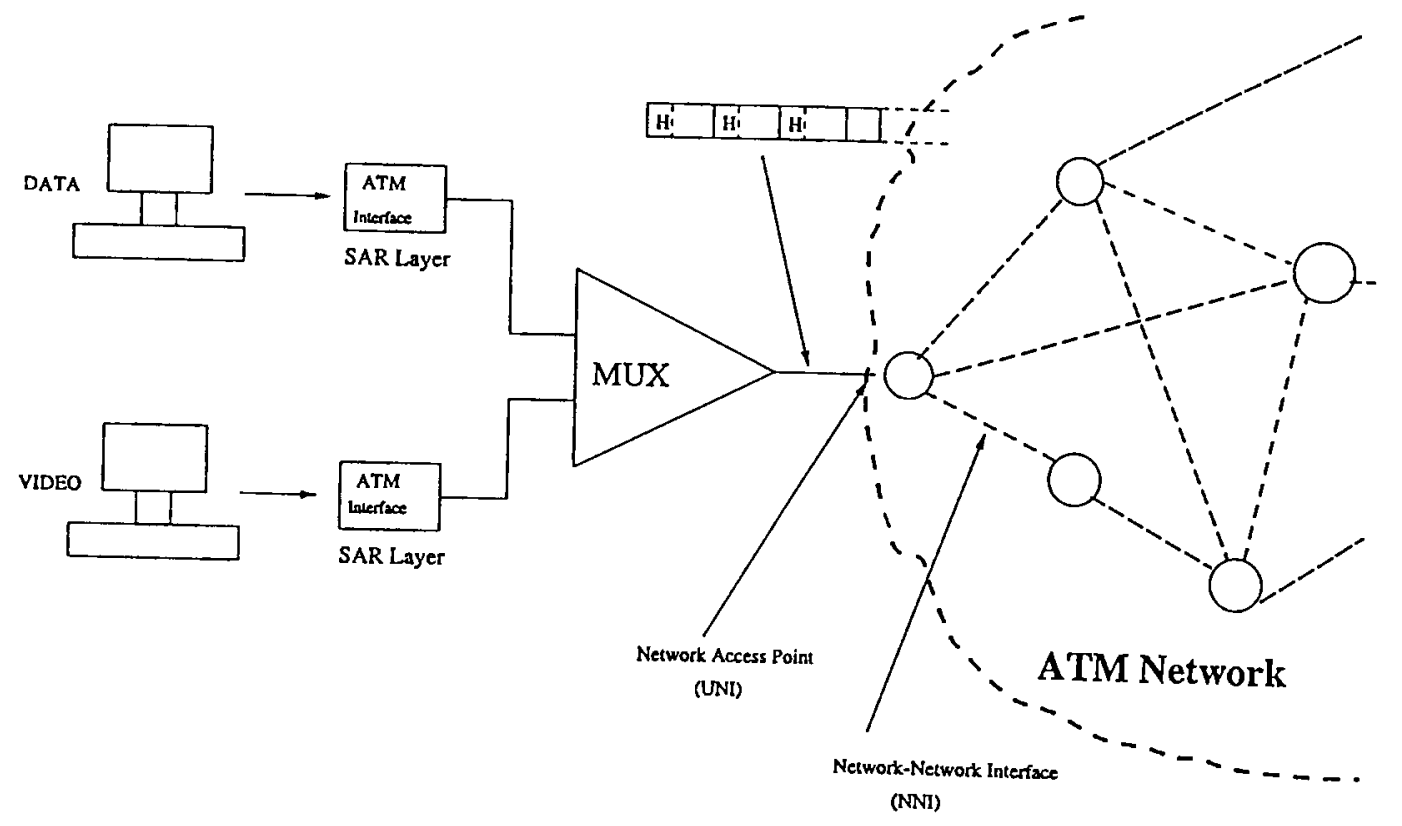

Fig. 1.1 ATM Aplication Example 


\subsection{Traffic Management and Related Issues in ATM}

ATM is not yet a reality in true sense. This is because of some unresolved issues related to traffic management. A large number of published technical articles describe the complexities, unsolved problems, issues, and proposed solution on the general topic of traffic management [12] [13] [14] [15]. The basic mechanisms for reserving bandwidth in ATM are fairly well defined by the Leaky Bucket Algorithm. The problem of achieving LAN-like flow and congestion control over ATM will take longer to solve and is a critical issue in the success of ATM. ATM forum is still reviewing the impact of delay and loss on applications. It is also verifying the feasibility of real-time critical Connection Admission Control (CAC). The solution will have to balance complexity against optimal performance and should achieve the acceptance of telephone and data communications industry. The problem of determining CAC procedures to implement a network to provide multiple QoS classes is imposing a fair amount of challenge. 
ATM is being jointly developed by two very different communities: the telephone community and the data communications community. Changing the telephone system to support new services requires a lot of time. Especially for large changes like replacing the interior of telephone network with ATM, the telephone community plans in terms of taking more than a decade to deploy a service. On this schedule, ATM would not be widely available in most countries before the year 2005 [19].

\subsection{Objectives of ATM}

A central objective in ATM networks is to provide virtual circuits that offer consistence performance in the presence of stochastically varying loads on the network [2]. This objective can be achieved in principle by requiring that users specify the traffic characteristics when a virtual circuit is established. This would allow the network to select a route that is compatible with the specified traffic and allocate resources as needed. Ideally a bandwidth management and congestion control mechanism should satisfy several objectives [2]. First, it should provide a consistent performance to those application that require it, regardless of the other virtual circuits with which a given virtual circuit may be multiplexed. Second, it should provide a high 
throughput even in the presence of the bursty traffic streams. (For a typical file transfer in LAN to LAN interconnect application, there may be more than 1000 ATM cells in the burst while the buffers in the switching systems will contain a room for typically 100 cells.) It is speculated that the success of ATM technology will depend on how well it can be used for the data transfer which is bursty inherently. Third, the specifications for the traffic characteristics should be simple enough to be easily understood by the end user. Inaccurate specifications in the QoS (Quality of Service) parameters by the user should not adversely affect the other users (connections). Finally it should be simple, flexible and economical.

\subsection{Review of the related work}

Many approaches to the bandwidth and buffer management problem have been proposed [2] and some of them are; Peak Rate Allocation, Minimum Throughput Allocation and Bursty Traffic Specification or Allocation. Boyer [10] suggested a "Fast Bandwidth Reservation Scheme" to handle bursty traffic with low peak rates. The scheme is based on "Fast Reservation Protocol" (FRP) which is designed to operate regardless of the upper layer protocol [12][8][10]. 
The source sends a request into the network along the virtual connection, asking permission to transmit a burst at a specified peak rate. All switches along the path examine the request and if the reserved bandwidth is available, they reserve the bandwidth and forward the request. If all the switches along the path accept the request, an acknowledgment is returned to the source from the far end. Before the burst is transmitted, a FRM (Fast Resource Management) cell which contains bit rate request on the affected connection along the path taken by the connection is sent. After receiving it, an attempt is made in every node to reserve the bit rate required for the transmission of the subsequent burst. If the bit rate can not be reserved on some link, a negative response (NAK) is returned immediately and the source can try later. If all nodes up-to and including the target node are able to provide the requested bit rate, a positive $\mathrm{ACK}$ is sent from target node to source node upon which the source can begin its transmission. Once the transmission has been completed, the reserved bit rate is released with the help of another FRM cell. FRP is a connection oriented multiplexing method that allows bandwidth negotiation at burst level. It assumes that CAC (Connection Admission Control) has been performed by other means. Turner [2] adopted a similar approach but applied it to a more difficult case of bursty traffic with peak rates 
that can be large fraction of link bandwidth. The approach treats burst itself as a request. If the bandwidth or buffer space is available for storing the burst, it is forwarded else it is completely discarded.

\subsection{Fast Buffer Reservation Protocol}

In this type of protocol a burst requests that buffer space be reserved in an intermediate node before it is admitted, as illustrated in Fig. 1.2. This can be done by an FRM cell at the beginning of the burst, by an AAL field, or by a predefined maximum burst length defined at the subscription time on a per VPI/VCI basis. The basic operation is that when a burst arrives at a node, a check is performed against the requested buffer (REQ BUF) size and the available buffer space at the node. If the requested buffer size is greater than the available buffer space, then the burst is not admitted; otherwise the entire burst is admitted. By ensuring that the entire burst can be buffered at a node, it can be guaranteed that no burst is ever lost, except through error or misinserted cells (unlikely events under normal circumstances). A higher layer protocol must detect if a burst was dropped, and retransmit it.

At present, the areas of Fast Resource Management (FRM) bandwidth and buffer reservation techniques are in the process of standardization. 


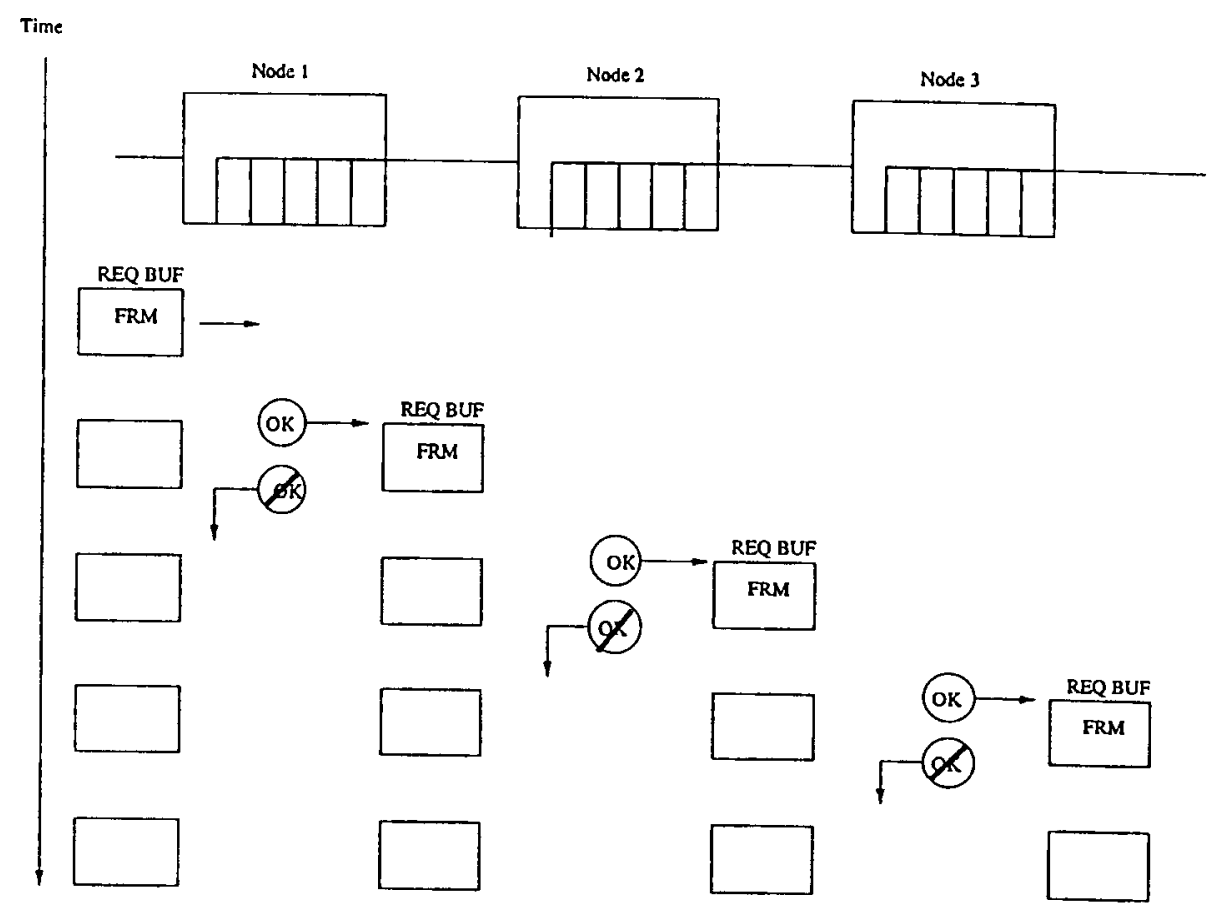

Fig. 1.2 Fast Buffer Management

\subsection{Overview of the thesis}

The effectiveness of the ATM lies in many facts, one of the most important is effective management of resources. The critical resources that can be managed are bandwidth and buffer space in the intermediate nodes. This becomes extremely important when bursty traffic is involved which demands 
for a specific amount of buffer reservations to store the bursts. The safe approach is to reserve the buffer space for each connection separately and independently, proportional to their peak rates only [2]. However this approach does not take the advantage of the fact that the bursty source will not be always active and thus will not need the reserved space all the time.

In this thesis, we extend the idea of Fast Buffer reservation and propose a method for efficient buffer allocation considering virtual circuit's peak rate, average rate and Source activity Probability (SAP). In chapter 2, we describe the proposed scheme. We also devise a Virtual Circuit Acceptance Algorithm.

Chapter 3 evaluates the performance of the scheme under various circumstances. The mechanism for Usage Policing is also described in this chapter.

In chapter 4 , we modify the scheme to include the multimedia traffic with different QoS requirements. We suggest an idea of partitioning the buffer space and evaluate its performance. The performance of partitioned scheme is compared with that of the non-partitioned.

Finally, chapter 5 concludes the thesis by discussing important finding of this thesis and outlining further research work. 


\section{CHAPTER 2}

\section{Proposed Scheme of Buffer Allocation for Bursty Traffic}

\subsection{Description and Mathematical Formulations}

In this scheme, we consider bursty traffic only. The user specifies peak rate $P R$ and average rate $A R$ for the virtual circuit. When a virtual circuit is active, it transmits at the rate $P R$ and when it is inactive, it does not transmit. The cell loss probability guaranteed by the network is fixed for the given network. The fast buffer allocation scheme [2] is used where the network reserves specific amount of buffers on each link (node) for a particular virtual circuit.

Let $L$ be the total number of buffer slots for the outgoing link and $R$ be the backbone link rate. (See Fig. 2.1) Note that $L$ buffer slots are shared in a common buffer pool. For any $i^{\text {th }}$ connection with peak rate $P R_{i}$, we need to reserve $B_{i}$ slots as given below [2].

$$
B_{i}=\left\lceil L \cdot P R_{i} / R\right\rceil
$$

where $\lceil x\rceil$ is the smallest integer greater than or equal to $x$. For example, for the first connection, the number of buffer slots reserved are given by $B_{1}=\left\lceil L \cdot P R_{1} / R\right\rceil$. However, as more connections come in, fewer slots will be 
reserved than actually needed according to (1). As an example, Say 10 buffer slots are required for the first connection according to (1). Then network

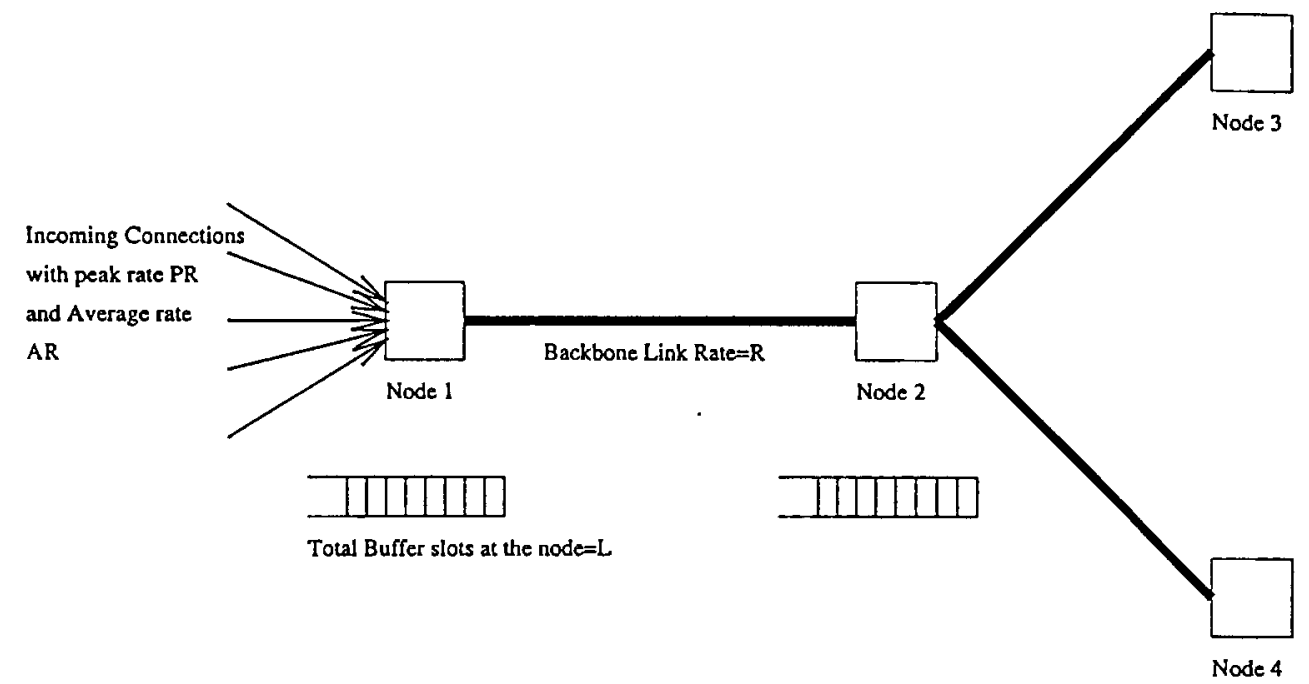

Fig. 2.1. Typical ATM Network 
reserves exactly 10 slots at the node in a common pool. Now suppose the second virtual connection requires 8 slots according to (1). Thus the total buffer requirement at the node is $10+8=18$, however the network reserves total number of slots less than 18. Say the network reserves 15 slots in total for the two connections (instead of 18) then the reduction factor $\alpha$ is equal to $\alpha=15 / 18$. The reduction factor is denoted by $\alpha_{i}$ where $\alpha_{i}$ is the value of $\alpha$ at the instant when $i^{\text {th }}$ connection is accepted. Thus, for the total of $n$ connections on the network, the total buffer slots reserved at the node is given by $\alpha_{n} \sum_{i=1}^{n} B_{i}$.

Let $x_{i}$ be the random variable describing buffer demand of the $i^{\text {th }}$ connection. i.e. $x_{i}=B_{i}$. Then it is reasonable to state that the minimum value of $\alpha_{n}$ will be

$$
\alpha_{n}(\min )=\sum_{i=1}^{n} x_{i} p_{i} / \sum_{i=1}^{n} x_{i}
$$

$$
1>\alpha_{n}>\alpha_{n}(\min )
$$

where $p_{i}=A R_{i} / P R_{i}$ denotes the probability that $i^{\text {th }}$ source is active. The value of $\alpha_{n}$ is adjusted at a level where cell loss probability is less than the threshold set by the network provider. Define a random variable $X$ representing the total buffer demand by all the virtual circuits. Then $X=\sum_{i=1}^{n} x_{i}$. 
Consider a link carrying $n$ virtual circuits with instantaneous buffer demands $x_{1}, x_{2}, x_{3}, \ldots \ldots, x_{n}$. The probability distribution for $\mathrm{X}$ can be most conveniently described using a moment generating function [2].

$$
f_{X}(z)=\prod_{i=1}^{n}\left(\bar{p}_{i}+p_{i} z^{B i}\right)=C_{0}+C_{1} z+C_{2} z^{2}+\ldots
$$

where, $\overline{p_{i}}=1-p_{i}$ and $C_{j}=\operatorname{Pr}(X=j)$ is the probability that the total buffer demand is $\mathrm{j}$. Let $k=\left\lceil\alpha_{n} L\right\rceil$. Then the cell loss probability is given by (3).

$$
P_{\text {loss }}=C_{k+1}+C_{k+2+\ldots}
$$

The $P_{\text {loss }}$ is calculated and it is made sure that it is less than a predetermined threshold. Note that, here we have reserved a common pool of the buffers of size $\alpha_{n} L$ instead of $L$ (assuming the network is fully loaded). The buffer space $\alpha_{n} L$ is a shared buffer space allocated to $n$ virtual connections. Let there be $\mathrm{H}$ hops through the connection. Then, total cell loss probability for the connection is given by [5]

$$
1-\left(1-P_{\text {loss }}\right)^{H} \approx H P_{\text {loss. }}
$$

\subsection{Virtual Circuit Acceptance Algorithm}

Whenever a connection is requested, the user specifies peak rate and average rate. For a given network, the cell loss probability bound (CLP Bound) is 
fixed. Assume there are $n$ connections on the network and user requests $n+1^{\text {th }}$ connection. The network keeps track of $\alpha_{n}(\min )=\sum_{i=1}^{n} x_{i} p_{i} / \sum_{i=1}^{n} x_{i}$ and from there it calculates $\alpha_{n+1}(\min )=\sum_{i=1}^{n+1} x_{i} p_{i} / \sum_{i=1}^{n+1} x_{i}$. For this new $\alpha_{n+1}(\min )$, the cell loss probability is calculated from (3) and compared with the threshold. If it is not acceptable, $\alpha$ which is equal to $\alpha_{n+1}(\mathrm{~min})$ is increased and CLP is recalculated. The whole process is repeated until cell loss probability is within the limit or $\alpha$ reaches 1 . The process is terminated when $\alpha=1$ and the new connection is rejected. The network provider may stop the process at some value of $\alpha$ which is less than 1 .

\subsection{Parameter Engineering}

The main parameters involved in this scheme are:
A. $\alpha$ for the first connection
B. $\alpha$ for subsequent connections.
C. cell loss probability threshold.

\subsection{1 $\alpha$ Reduction Factor}


The value of $\alpha$ plays an important role in this scheme as it decides the effectiveness of the scheme. It is closely related to the CLP bound guaranteed by the network provider. For the first connection, $\alpha$ is set to 1 (i.e. $\alpha_{1}=1$ ). Thus, there are no savings in the buffer space for the first connection. However, as more connections come in, advantage of their bursty characteristic is taken and $\alpha$ changes from 1 to some value between 1 and $\alpha(\min )$ as given in (2). In the simulation model, it is seen that generally $\alpha(\mathrm{min})$ is not sufficient for the given cell loss probability. In the best scenario the value of $\alpha$ will be $\alpha$ (min). In the worst scenario $\alpha$ is equal to 1 . When it becomes 1 , apparently there are no savings gained in this scheme. However, the probability of having $\alpha$ be 1 in case of bursty sources is very low.

\subsubsection{Cell Loss probability threshold}

This is to be decided by the network provider. If this threshold is selected to be very low, then $\alpha$ is very close to 1 . In this case the savings obtained are not very significant. However, it is observed that the savings in the buffer space increases when the number of connections increases or $p_{i}$ reduces with peak rate remaining the same or threshold of cell loss probability (CLP bound) 
increases. Again, note that the cell loss probability threshold is to be fixed for the network and all the virtual circuits will be guaranteed that or better.

\subsubsection{Tariff}

This scheme provides a simple means for charging the user. The user is charged according to $\alpha$ prevailing in the network and the peak rate specified by the user. The tariff is reduced as more users gain the access to the network. For homogeneous connections, which is the case considered here, users can be charged according to $\alpha$ only. However, for heterogeneous connections, it is required to consider $\alpha, p_{i}$ and peak rate. 


\section{CHAPTER 3}

\section{Performance Analysis of the Proposed Scheme}

\subsection{Performance of the Single Buffer Scheme}

The basic aim of this scheme is to maximize the efficiency of the network regarding buffer space allocation as compared to Turner's scheme where $\alpha$ is equal to 1 .

We simulated this scheme in $\mathrm{C}$ programming language. In the simulation model, we assumed that all the connections are homogeneous and independent of each other. This means they have the same peak rate and average rate with the same buffer requirement. The results of simulation for cell loss probability of 1.5E-3 are as shown in Fig. 3.1. It is seen from the results that as the $p_{i}$ is increased from 0.1 to 0.6 with number of connections i.e. $\mathrm{N}=10$, the saving gained in buffer space reduces from approximately $50 \%$ to $0 \%$. The relationship between the $p_{i}$ and "saving in buffer" is not quite linear for $\mathrm{N}=10$.

However, our study indicates that as $\mathrm{N}$ increases, the percentage savings goes up for a given $p_{i}$ and the "savings curve" becomes linear. This phenomenon suggests that for a large number of connections, say 100 , (which could be typical 
case for an ATM network), we can estimate the percentage savings from $p_{i}$ using some empirical relationship.

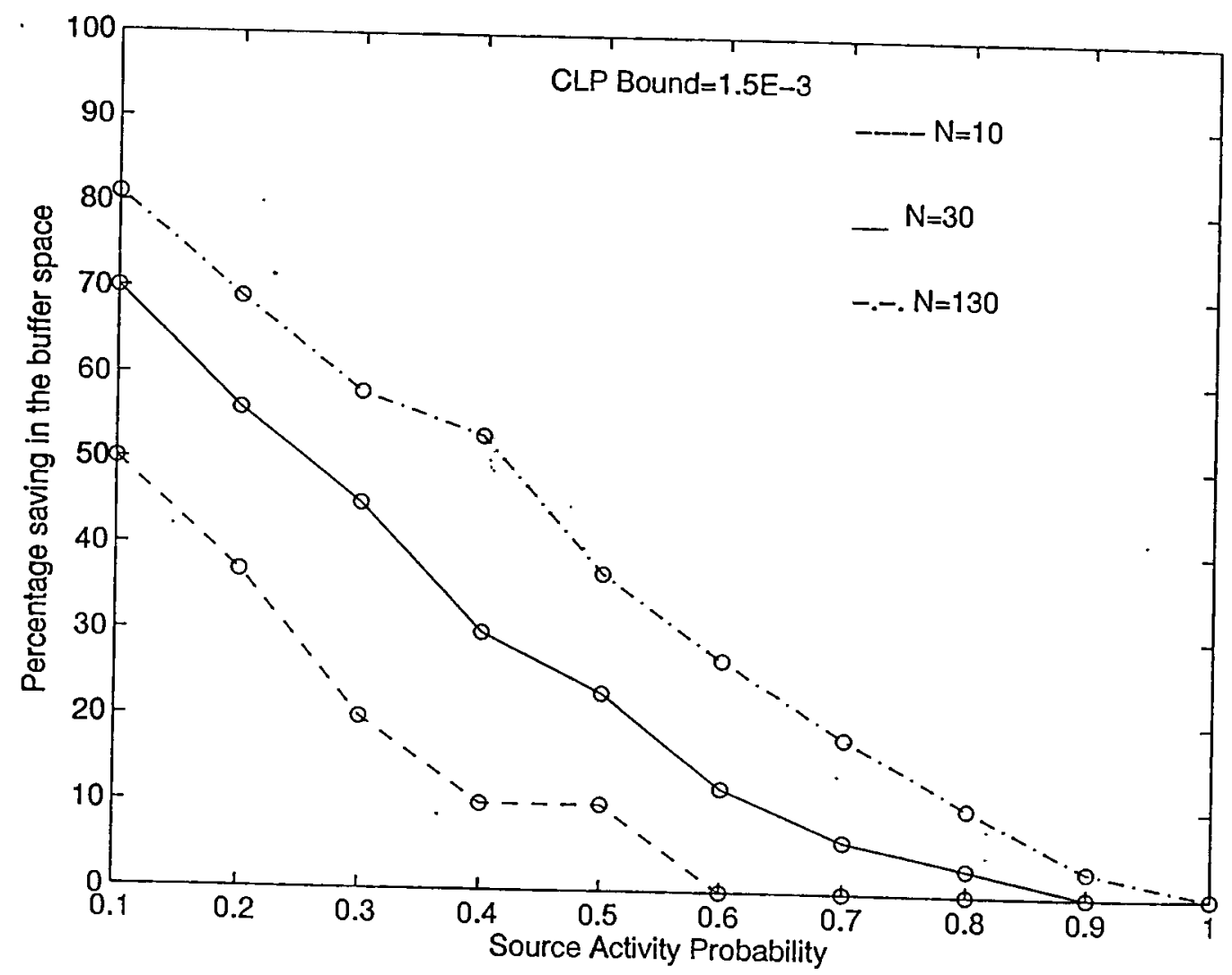

Fig. 3.1 Saving in the buffer space with CLP=1.5E-3. 
As file transfer applications are more "loss sensitive" than "delay sensitive" we ran the simulation for CLP=1.5E-4 and obtained the results as shown in Fig. 3.2. The results show a slight reduction in the savings of buffer as compared to the previous results. Also the relationship between SAP and savings in the buffer space tend to become linear with increase in number of network connections $(\mathrm{N})$. This suggests that the savings will reduce as the network will become stringent regarding its CLP bound. From the results we can see that for $p_{i}=0.4, \mathrm{~N}=30$, the savings of the buffer are still $28 \%$. However, if we consider the case of $p_{i}=0.5, \mathrm{~N}=30$ for two different CLP bounds (1.5E-3 and 1.5E-4), we can obtain savings up to $25 \%$ and $18 \%$ respectively. This also leads us to believe that for a large number of connections the CLP bound has very little effect on the percentage savings of buffer space. Note here that saved buffer space indicates more number of connections that a network can accept thus increasing the network revenue. This will also help to keep tariff low and make ATM a reality. The simulation results for CLP bound of 1.5E-7 are as shown in Fig. 3.3.

\subsection{Usage Policing Mechanism}


In this section, we propose a policing scheme for the bursty traffic. It may be noted that, the scheme requires that a violating connection should be thrown out of the network immediately as it will adversely affect the other connections.

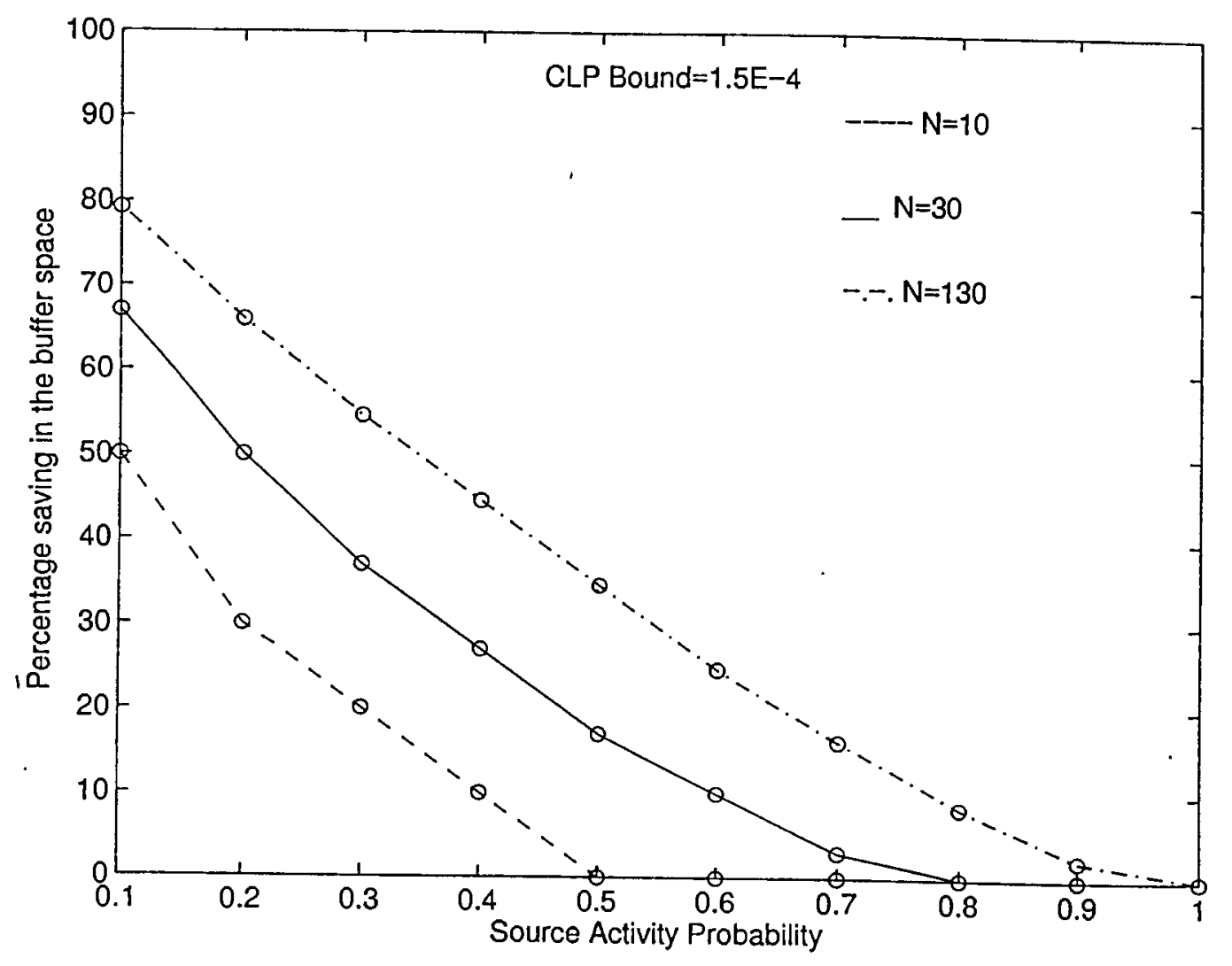

Fig. 3.2 Saving in the buffer space with CLP=1.5E-4. 


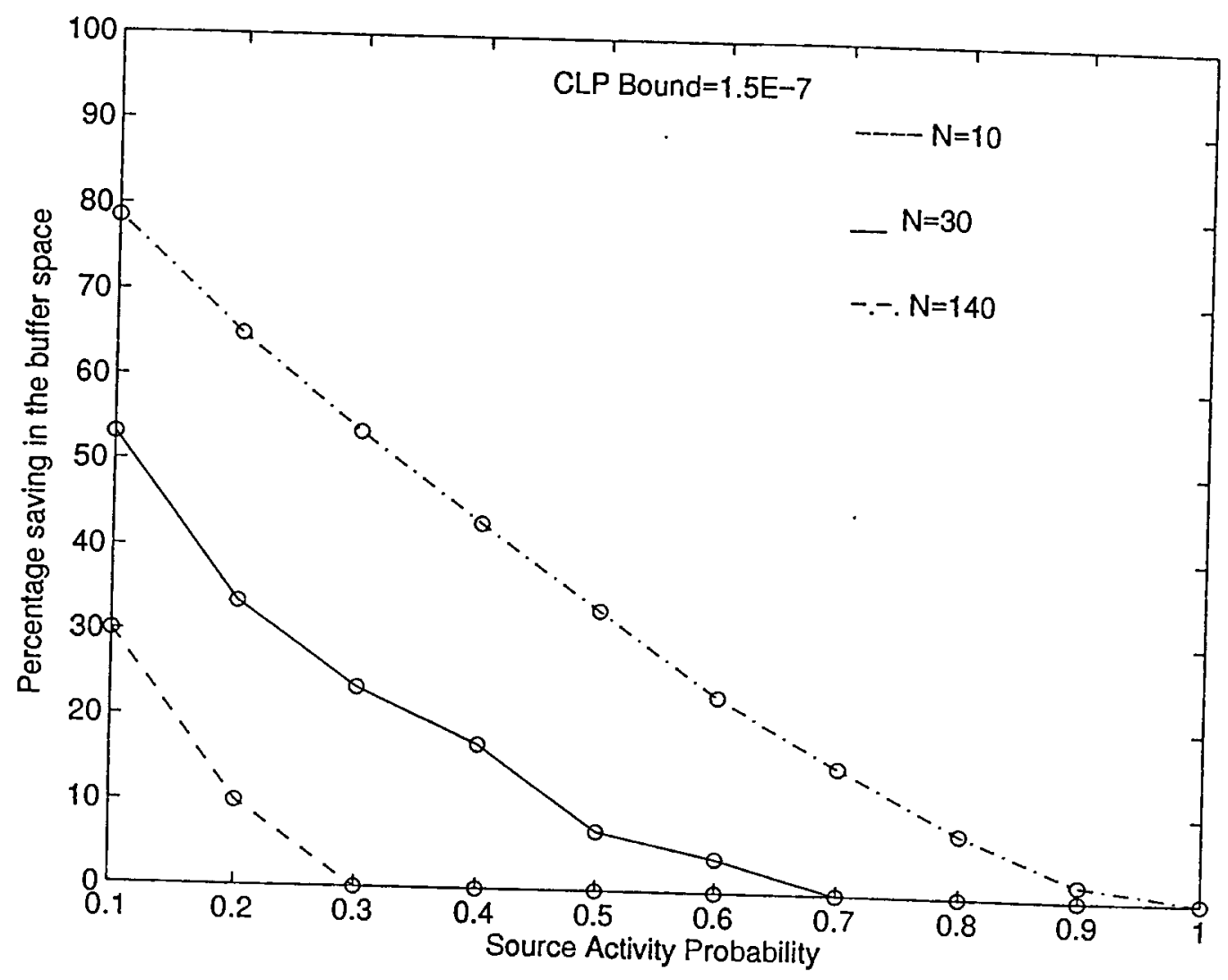

Fig. 3.3 Saving in the buffer space with $C L P=1.5 E-7$. 
This is due to the fact that the buffer space is a shared common pool. If any connection violates a traffic contract, it will need (occupy) more buffers leaving fewer buffers for the other connections.

When a user requests a connection, the service contract is defined. The user specifies both the peak rate and the average rate for the connection. No user once accepted in the network is allowed to exceed any of these specifications. Strict action has to be taken if such violation is cited. The hardware implementation for the usage policing is as shown in Fig. 3.4.

The clock period can be adjusted to "one unit time" and the threshold can be set at $P R_{i}$. At the beginning of burst, i.e. the start of burst cell, the counter is incremented as each cell comes in. Any time (within the unit time), when the counter exceeds threshold, the following cells are discarded and the connection is declared as a "violating" connection. If the end of burst cell is received within the clock period, the counter is reset to 0000 and prepares for the next burst. After "one unit time", the counter is automatically reset to 0000 and it is incremented from the next cell onwards. This is shown in Fig. 3.5.

Note that careful consideration should be given to the clumping effect of CDV (Cell Delay Variation) when policing the connection [5]. If CDV is not considered, cells may appear to be transmitted at higher rate than specified, 


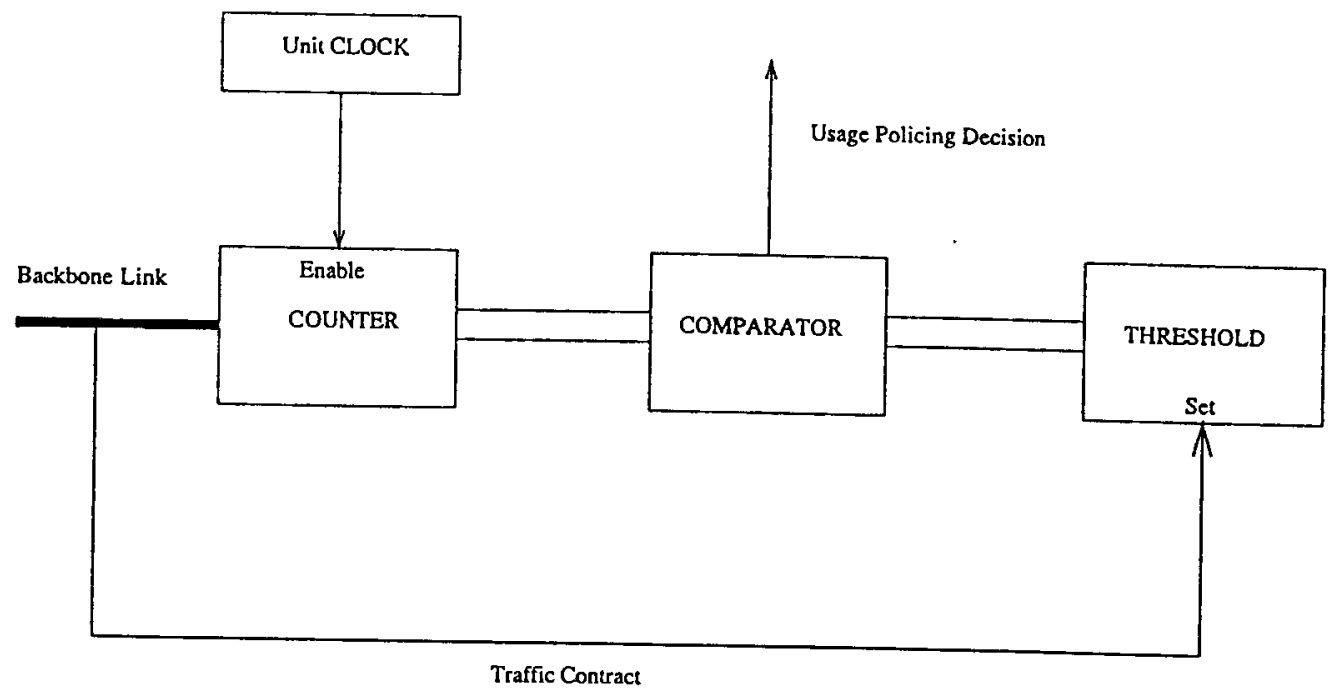

Fig. 3.4. Usage Policing Mechanism. 


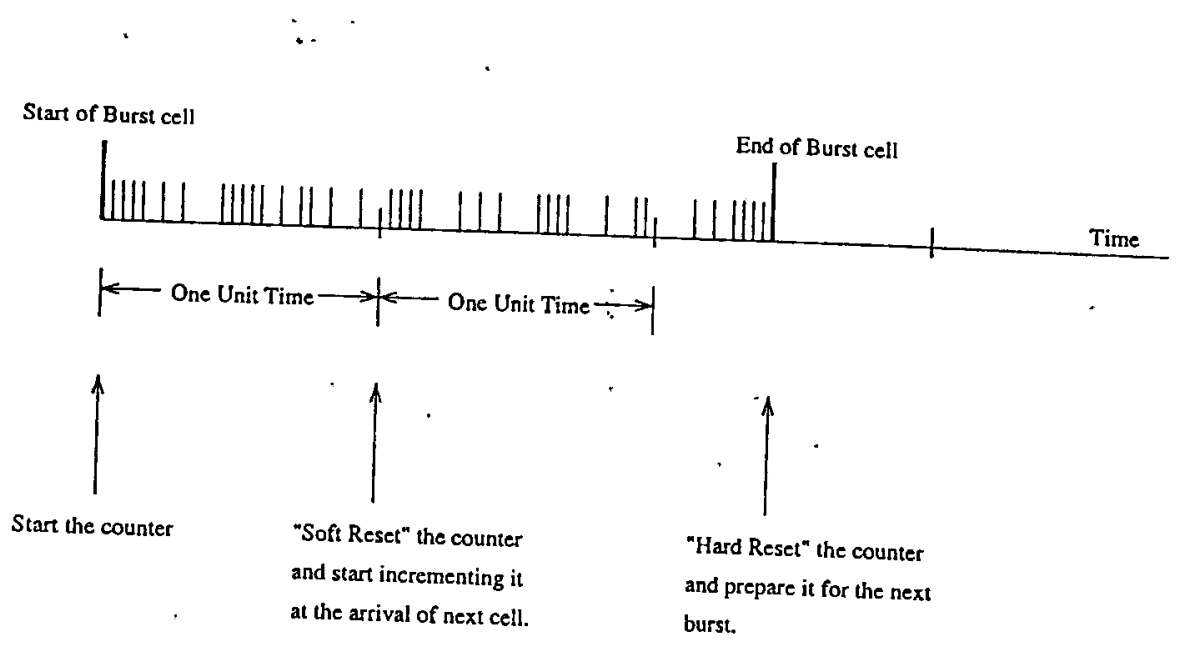

Fig. 3.5 Usage Policing Events in ATM Networks. 
although the user is not actually violating the traffic contract. For considering $\mathrm{CDV}$, the unit clock time of policing may be adjusted in the following way.

Policing Time $=$ Actual unit Time $-\mathrm{CDV}$ of the network.

We suggest that the monitoring time should be kept as low as possible because of following advantages.

A. The need of large bit counter can be avoided.

B. Quick and prompt indication of Usage violation is possible.

Also the delay between the following events should be kept minimum to prevent underpolicing when peak rates of the connection are high.

A. Resetting the counter to 0000 at the end of unit clock time.

B. Incrementing the counter from next cell.

If the peak rate of the connection is high, the UPC counter will miss some of the cells before it starts incrementing the counter. This will lead to Underpolicing of the connection. The end result would be degradation in QoS for all the connections when network is fully loaded.

3.3 Fig. 3.6 shows a modified Usage Policing Mechanism by adding an additional counter associated with a single virtual connection. The Enable pulses have the ON period of $\mathrm{T}=$ Usage Policing Time $-\mathrm{CDV}$. The inverter allows 
smooth and fast transition from Counter 1 to Counter 2 thus avoiding Underpolicing.
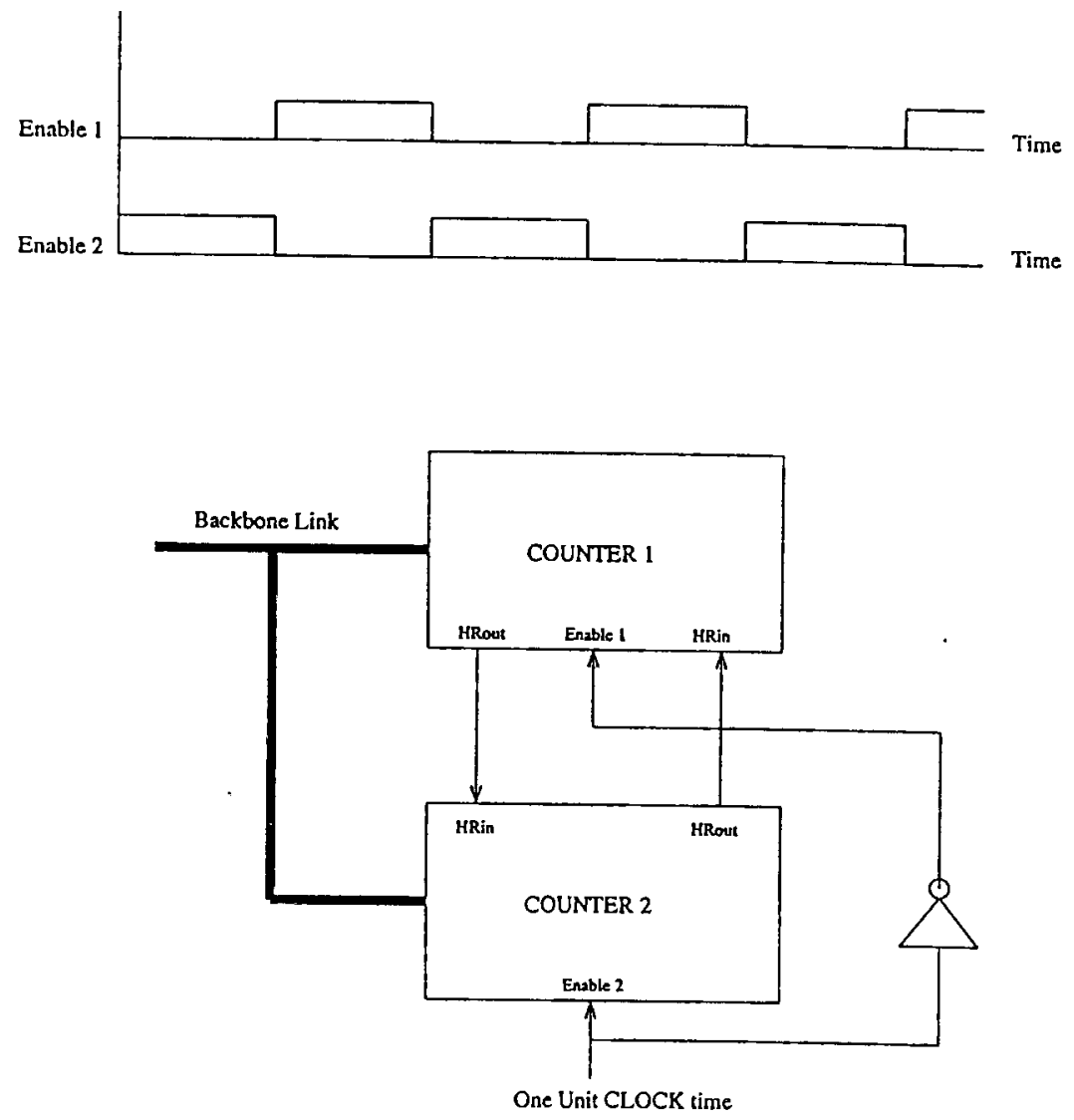

Fig. 3.6 Modified Usage Policing Scheme. 


\subsection{Inadequacies of Single Buffer Allocation Scheme}

In the previous section, we examined the performance of the scheme when a network supports only one CLP bound; i.e. $1.5 \mathrm{E}-3$ or $1.5 \mathrm{E}-4$ or $1.5 \mathrm{E}-7$. However, this is clearly not acceptable as it seriously impairs the ability of the network to support the multimedia traffic with different QoS requirements.

The solution to this problem can be found by partitioning the total buffer space into a number of different service classes supported by the network which is discussed in the next chapter. 


\section{CHAPTER 4}

\section{Partitioned Buffer Allocation Scheme}

\subsection{Description}

In the previous chapter, we examined the performance of the scheme when a network supports only one CLP bound; i.e. $1.5 \mathrm{E}-3$ or $1.5 \mathrm{E}-4$ or $1.5 \mathrm{E}-7$. However, this is clearly not acceptable as it seriously impairs the ability of the network to support the multimedia traffic with different QoS requirements.

The solution to this problem can be found by partitioning the total buffer space into a number of different service classes supported by the network. Here we assume two service classes (as specified) [9].
A. Class 1 with CLP bound of 1.5 E-4 (Typical file transfer.)
B. Class 2 with CLP bound of 1.5 E-7 (Video/ Interactive data.)

For simplicity, we assume that the buffer space is equally partitioned between these two services. However, different proportions of partitioning could also be used. We simulated this and obtained the following results. As mentioned before, the saving gained in the buffer space is greatly dependent on the number of connections the network generally supports with available buffer space. As we partition the buffer space, the number of connections in each class of service that 
a network can support is reduced to half. In this paper, again for simplicity, we assume that all the connections are identical in their QoS requirements. Also it is assumed in the analysis that the network is $100 \%$ loaded by bursty traffic. Without implementing this scheme, the traffic needs all the buffer space at the node. We observed that a significant amount of savings in buffer could be obtained if the number of connections supported by the network is high. There are three ways to configure the buffer space.
A. Single Buffer with CLP bound of 1.5 E-4 guaranteed by the network.
B. Single Buffer with CLP bound of 1.5 E-7 guaranteed by the network.
C. Partitioned buffer with two independent service classes as described above.

\subsection{Performance Comparison of Partitioned Buffer Scheme}

We simulated the partitioned buffer and obtained the amount of savings under circumstances.

\subsubsection{Single Buffer with CLP bound of $1.5 \mathrm{E}-4$ guaranteed by the network.}


In this case, the network can not accept any connection that needs CLP better than $1.5 \mathrm{E}-4$. Though the savings in this case are high, network loses the services that require lesser CLP bounds. Thus, this is not a practical approach.

\subsubsection{Single Buffer with CLP bound of 1.5 E-7 guaranteed by the network.} Here network guarantees "At Best" service (CLP=1.5E-7) even if connection does not require it. The percentage savings in buffers are less as compared to previous case (See Fig. 3.2 and Fig. 3.3.) However, as the number of connections supported by the network increases, the scheme performs much better. As $\mathrm{N}$ reduces, the savings reduce drastically.

\subsubsection{Partitioned buffer with two independent service class space}

The total number of connections the network can support remains the same. However, the maximum number of connections in class 1 or class 2 reduce to half. If network receives all class 1 or class 2 connection requests, this scheme fails to perform well. On average the buffer savings in this scheme are less than savings in A or even in B as shown in Fig. 4.1.

\subsection{Results of Comparison}


The simulation results provide us with some important results. Firstly, a single buffer scheme (SBS) with network CLP bound of 1.5E-4 yields higher savings than that with CLP bound of 1.5E-7. However, if employed, it will prevent the network from accepting the connections that need CLP better than 1.5E-4.

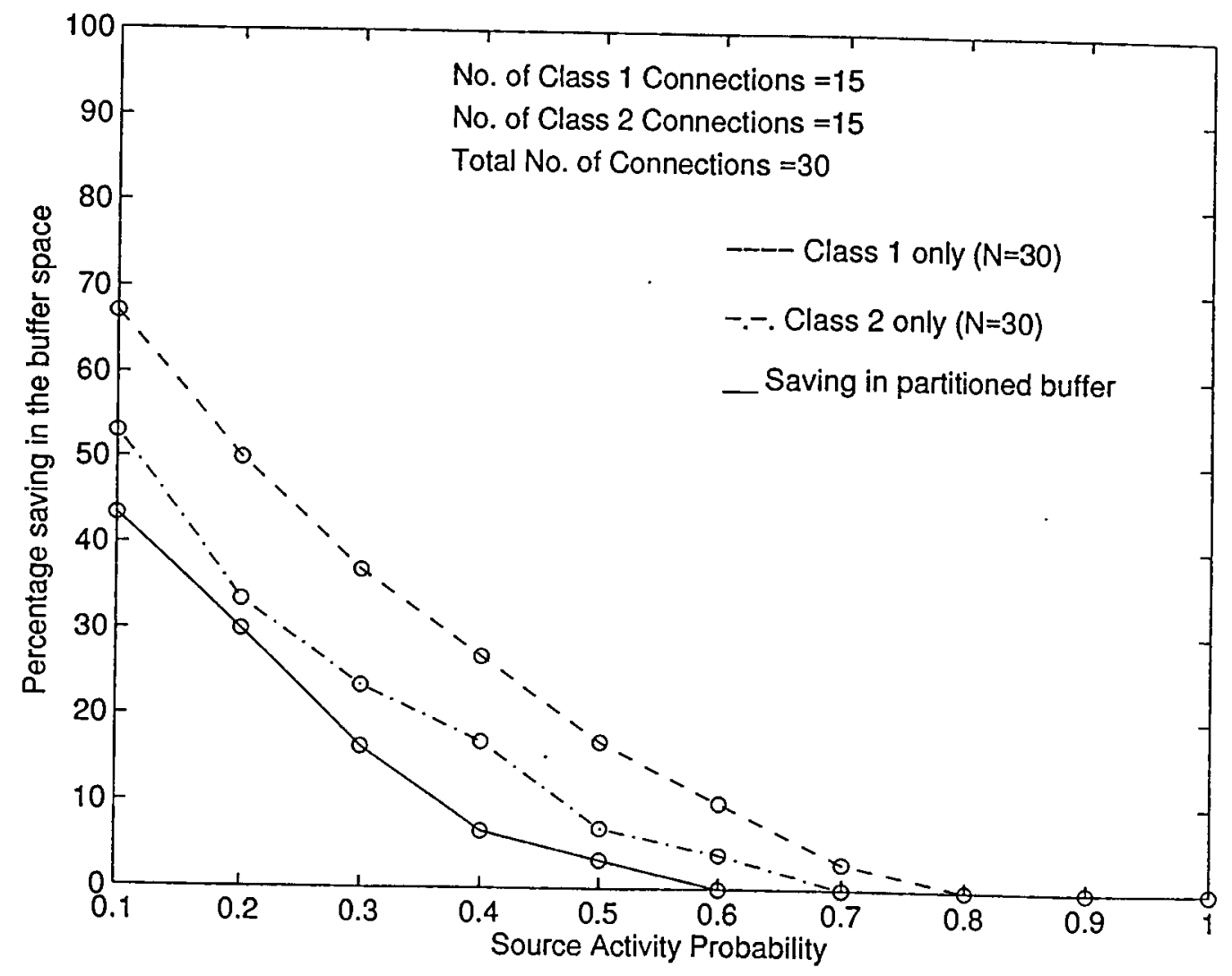

Fig. 4.1 Class 1, Class 2 and Average Buffer Saving 
The SBS with CLP 1.5E-7 eliminates this problem however apparently it seems to be waste of resources. This is because the services not needing so tight CLP will also be assured that.

The partitioned buffer scheme (PBS) with two independent service classes provide less savings than that in A or B. This is because partitioning reduces the maximum number (when network is fully loaded) of connections that a network can accept by $50 \%$.

Thus, essentially, the savings with PBS are less compared to SBS with tightest network CLP bound. 


\section{CHAPTER 5}

\section{Conclusion And Research Findings}

\subsection{Discussion}

In this thesis, we proposed a modified buffer allocation scheme for the bursty traffic in ATM networks. The scheme is based on Fast Reservation Protocol suggested by Boyer [10]. We considered all the connections to be bursty (SAP low) and independent of each other. The simulation results were found for different network CLP bounds. i. e. 1.5E-3, 1.5E-4, 1.5E-7. As the basic theme of the scheme is "Common pool of buffers", it is important that a user violating the service contract should be thrown out of the network. Thus usage policing is very crucial in this scheme. Therefore we also suggested a suitable Usage Policing Mechanism. The effect of CDV on UPC was also considered. As ATM network should also support multimedia services, we studied the effect of partitioning the buffer space according to service classes. The specific research findings are discussed in the next section.

\subsection{Important Research Findings.}

It was observed that for Single Buffer Scheme (SBS), the savings in the buffer depend on network CLP bound, Source Activity Probability and No. of 
connections prevailing in the network. For a given CLP bound, as SAP increases, the savings go down. Also for a given SAP, savings in the buffer space reduces as network CLP bound reduces. As N is increased, the effect of network CLP bound on savings in the buffer space reduces. The asymptotic behavior ,however, was observed with savings when $\mathrm{N}$ is increased. Thus for large $\mathrm{N}$, the effect of CLP bound is negligible on savings of buffer. As ATM network must support multimedia, we considered various service classes with different CLP requirements. To accommodate this traffic, the partitioning of buffer was studied. It is seen that on average the partitioned buffer scheme (PBS) leads to lesser savings than that in SBS with tightest CLP bound requirement.

\subsection{Some drawbacks in the Scheme and Solutions}

The proposed scheme is an extension of the Fast Buffer Reservation Scheme [2] which seems to perform well in case of bursty traffic. The network throughput and hence the revenue of the vendor could be increased with this scheme. However there are some drawbacks to this scheme.

First, imagine a "live" video transmission of the same event. As all the virtual connections are carrying the same data, they will be "Active" or "Inactive" at the same time. Thus there will be always a conflict for buffer space and some 
connections will experience seriously degraded service. In this situation, the underlying assumption of "independent" connections is no longer true.

Secondly, the first connection will be charged more if it is the only connection on the network as for the first connection is 1 . Therefore every user will try to use the network during "Rush Hours" thus increasing the rush during that time. Then more connections are likely to be rejected because of unavailability of the resources. Also during "cold" hours the network will remain underloaded while during the "hot" hours it will be overloaded. However this problem can be solved by charging "less" during economy times. Also a more complicated model for tariff could be used.

Thirdly, the QoS guaranteed by the network is fixed in this scheme. User has no control over it. However this problem can be solved by partitioning the buffer space as mentioned before.

Finally, the assumption that all connections are homogeneous and independent gives us some optimistic results. We envision that in situations where these assumptions are no longer valid, the results will be degraded by $10 \%$ to $15 \%$ depending on the $\mathrm{N}$. However, in the light of situation where $\mathrm{N}$ is large, these assumptions have a very little effect on our results. 


\subsection{Future Research}

The partitioning suggested in this thesis is very rigid. We considered only equal partitioning with two service classes. However, in true ATM environment this is clearly unacceptable if we have to accommodate multimedia. Also the savings obtained in SBS or PBS are conditional i. e. only when the network is fully loaded. This assumption provides us some optimistic results regarding buffer savings. In this research, we did not consider the effects of partially loaded network on buffer savings. The author hopes that the basic scheme developed here, could be extended to incorporate such effects.

Further research can be carried to find out optimum partitioning in order to maximize the buffer savings in certain situation. The effect of "Adaptive Partitioning" can also be studied in various traffic pattern environments. At the same time, it is necessary to investigate the cost of implementation. There is a tradeoff between the complexity of the network and savings obtained. It is important to find out the balance between the two to prevent the prohibitive cost of implementation. 


\section{GLOSSARY OF SOME TERMS IN THIS THESIS}

Asynchronous Transmission - The transmission of data through start and stop sequences without the use of common clock.

Asynchronous Transfer Mode - A high speed connection-oriented multiplexing and switching method specified in international standards utilizing fixed length cells to support multiple types of traffics. It is asynchronous because cells carrying user data need not be periodic.

ATM Adaptation Layer (AAL) - A set of internationally standardized protocols and formats that define support for circuit emulation, packet video and audio, and connection-oriented and connectionless data services.

Broadband - A term that refers to channels supporting rate in excess of DS3 (45 Mbps) or E3 (34 Mbps).

Broadband ISDN (B-ISDN) - A set of services, capabilities and interfaces supporting an integrated network and user interfaces at speeds greater than that of ISDN. The ITU-T initially decided to develop B-ISDN using ATM in 1988.

Cell - A fixed length 53 octet packet or Protocol data Unit (PDU) used in ATM. It has a 5 byte header and 48 byte payload. 
Cell Delay Variation (CDV) - The measure of cell clumping, which is heuristically how much more closely the cells are spaced than the nominal interval.

Cell header - A 5-octet header that includes control information used in processing, multiplexing and switching cells.

Congestion - The condition where network resources (bandwidth and buffer) are exceeded by an accumulation of demand.

Connection Admission Control (CAC) - The set of procedures to be carried out by the network at Network Access Point to ensure that the available resources will not be exceeded by the connection if it is accepted. CAC decides whether to accept the connection or not depending on resources available and requested by the service.

Forward Explicit Congestion Notification (FECN) - Convention in frame relay for a network device to notify the user device that network congestion is occurring.

Local area Network (LAN) - A MAC level data and computer communications network confined to short geographic distances.

Media Access Control (MAC) - IEEE 802 defined media specific access control protocol. (See also $L A N$ ) 
Permanent Virtual Circuit (PVC) - A logical dedicated circuit between two user ports in a point to point configuration.

Service Access Point (SAP) - The access point at the network node or station where the service users access the services offered by service providers. (Also called NAP (Network Access Point))

Switched Virtual Circuit - Virtual circuits similar to PVCs but established on a call-by-call basis.

Synchronous Optical Network (SONET) - A U. S. High speed fiber optic transport standard for fiber optic digital hierarchy (speeds range from $51.84 \mathrm{Mbps}$ to $2.4 \mathrm{Gbps})$

Transmission Control protocol/Internet Protocol (TCP/IP) - The combination of a network and transport protocol developed by ARPANET for internetworking IP-based networks.

Usage Parameter Control (UPC) - The policing method that monitors the resource usage by the admitted connection and makes sure that the traffic contract is not violated. If violation is cited, UPC may just tag the non-conforming cells or simply discard them.

User-to-Network Interface (UNI) - The point where the user accesses the network. 
Virtual Channel Identifier (VCI) - In ATM, a field within the cell header which is used to switch virtual channels.

Virtual circuit - A virtual connection established through the network from origination to destination, where packets, frames, or cells are routed over the same path for the duration of the call. These connections seem like dedicated paths to the users, but are actually network resources shared by all users. Bandwidth on a virtual circuit is not allocated until it is used.

Virtual Path Identifier (VPI) - In ATM, a field within the cell header which is used to switch virtual paths, defined as groups of virtual channels. 


\section{REFERENCES}

[1] ITU Recommendations, I series (B-ISDN), July 1992.

[2] Jonathan S. Turner, "A Proposed Bandwidth Management and Congestion

Control Scheme for Multicast ATM Networks", WUCCRC-91-1. Washington University at St. Louis, Computer And Communications Research Center Report.

[3] S. E. Minzer, "Broadband User-Network Interfaces to ISDN", Proc. ICC '87,Seattle, June 1987, pp. 363-369.

[4] A. Gersht and K. J. Lee, "A Congestion Control Framework for the ATM Networks", IEEE JSAC vol. 9, pp. 1119-1130, September 1991.

[5] Naoaki Yamanaka, Youichi Sato and Ken-ichi Sato, "Usage Parameter Control and Bandwidth Allocation Methods Considering Cell Delay Variation in ATM Networks", IEICE Trans. Comm.,vol. E76-B, No. 3, pp. 270-278, March 1993.

[6] Peter Newman, "Traffic Management for ATM Local Area Networks" IEEE Communications. Mag., pp. 44-50, August 1994.

[7] J. W. Roberts, "Traffic Control in the B-ISDN" Computer Networks and ISDN systems, pp. 1055-1063, 1993. 
[8] P. E. Boyer, "Congestion Control for ATM", Int'l Teletraffic Congress Seminar on Broadband Technologies: Architectures, Applications and Performance. 10/90.

[9] Gillian M. Woodruff and Rungroj Kositpaiboon, "Multimedia Traffic Management Principles for Guaranteed ATM Network Performance", IEEE $J S A C$, vol. 8, No. 3, pp. 437-446, April 1990.

[10] P. E. Boyer and D. P. Tranchier, "A Reservation Principle with Applications to the ATM Traffic Control", Computer Networks and ISDN Systems, vol. 24., pp. 321-334, 1992.

[11] Walfgang Fischer, Eugen Wallmeier, Thomas Worster, Simon P. Davis and Andrew Hayter, "Data Communication using ATM: Architectures, Protocols and Resource Management”, IEEE Communication Mag., pp. 24-33, August 1994.

[12] C. Lea, "What Should be the Goal for ATM?", IEEE Network, Septembet 1992.

[13] M. Wernik, O. Aboul-Magd, H. Gilbert, “Traffic Management for B-ISDN services," IEEE Network, September 1992.

[14] M. Decina, “Open Issues Regarding the Universal Application of ATM for Multiplexing and Switching in the B-ISDN," ICC 1991.

[15] R Binder, "Issues in Gigabit Networking," Globecom, 1992. 
[16] D. E. McDysan and D. L. Spohn, ATM Theory and Application, McGrawHill, 1994.

[17] M. De Prycker, Asynchronous Transfer Mode: Solution for Broadband ISDN, 2nd Edition, Ellis Horwood, 1993.

[18] ATM Forum, ATM User-Network Interface Specification Version 3.0, August 1993.

[19] Craig Patridge, Gigabit Networking, Addison-Wesley, 1993. 